\title{
To Be or Not To Be ... Male
}

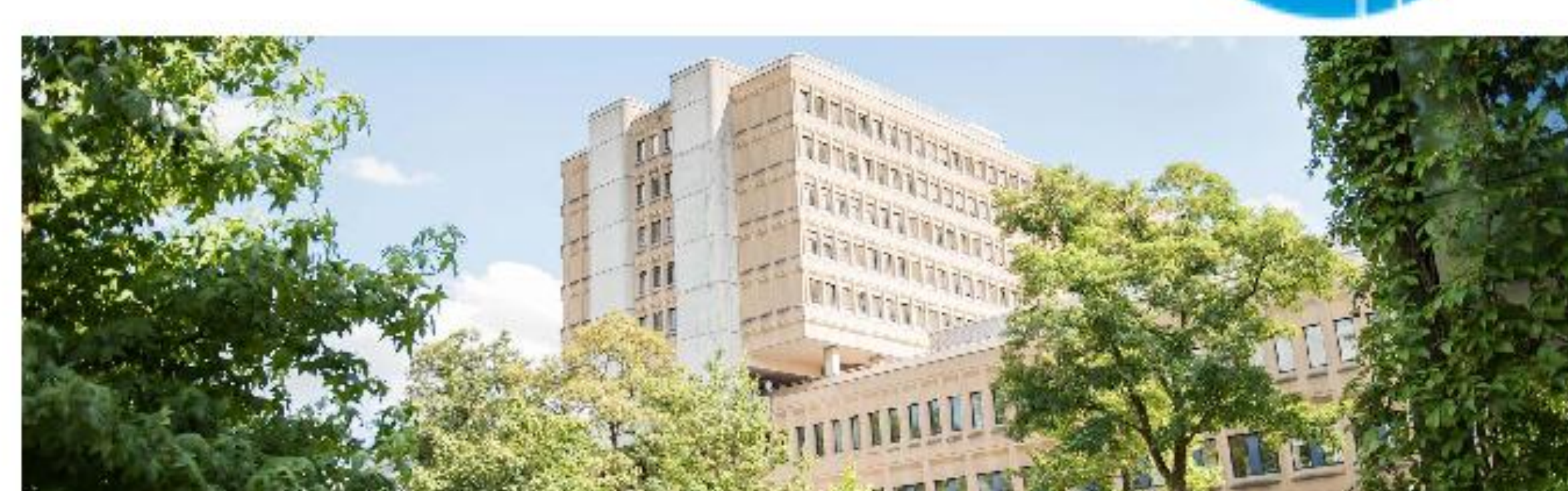

Capraro J (1), Schütz P (1)

(1) Division of Endocrinology, Diabetes and Metabolism, Kantonsspital Aarau, Switzerland

\section{Introduction:}

Pats. with Klinefelter Syndrome (KS) have elevated morbidity and mortality due to several reasons. Yet, there is no connection between $\mathrm{KS}$ and male-to-female (MtF) sex change.

\section{Case Report}

A 74-year old man was sent for endocrine work-up prior to surgery due to gynecomastia. The patient had lived as a MtF transgender for many years. He was told not to qualify for a transgender surgical approach when he was 55 years old. He took estradiol substitution for several years, but by age 60 after developing recurrent severe pulmonary embolism he stopped substitution. After that he lived "asexual" and bilateral, non-painful gynecomastia developed (or became disturbing). Examination revealed small testes (Picture 1), bilateral Gynecomastia (Tanner IV, Picture 2) and signs of chronic venous insufficiency. The biochemical analyses showed hypergonadotropic hypogonadism with otherwise normal values. The molecular analyses revealed a 47XXY Karyotype. Osteodensitometry showed low peak bone mass (Picture 3 ). We started topical testosterone replacement and calcium/vitamin D3 substitution.

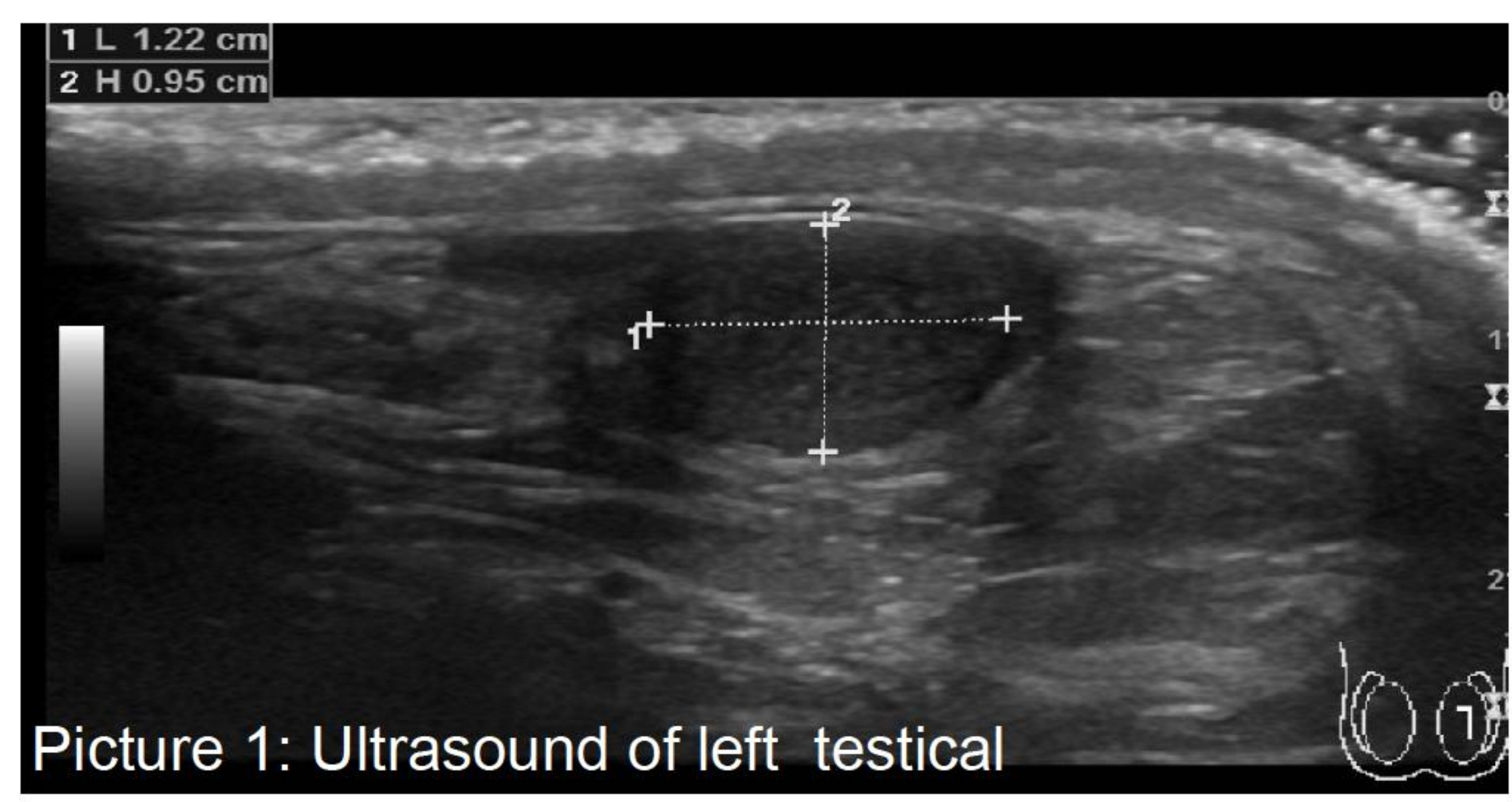

Fig 1: HORMONAL IRREGULARITIES IN MTF (AFTER AUER, FERTILE STERIL 2013)

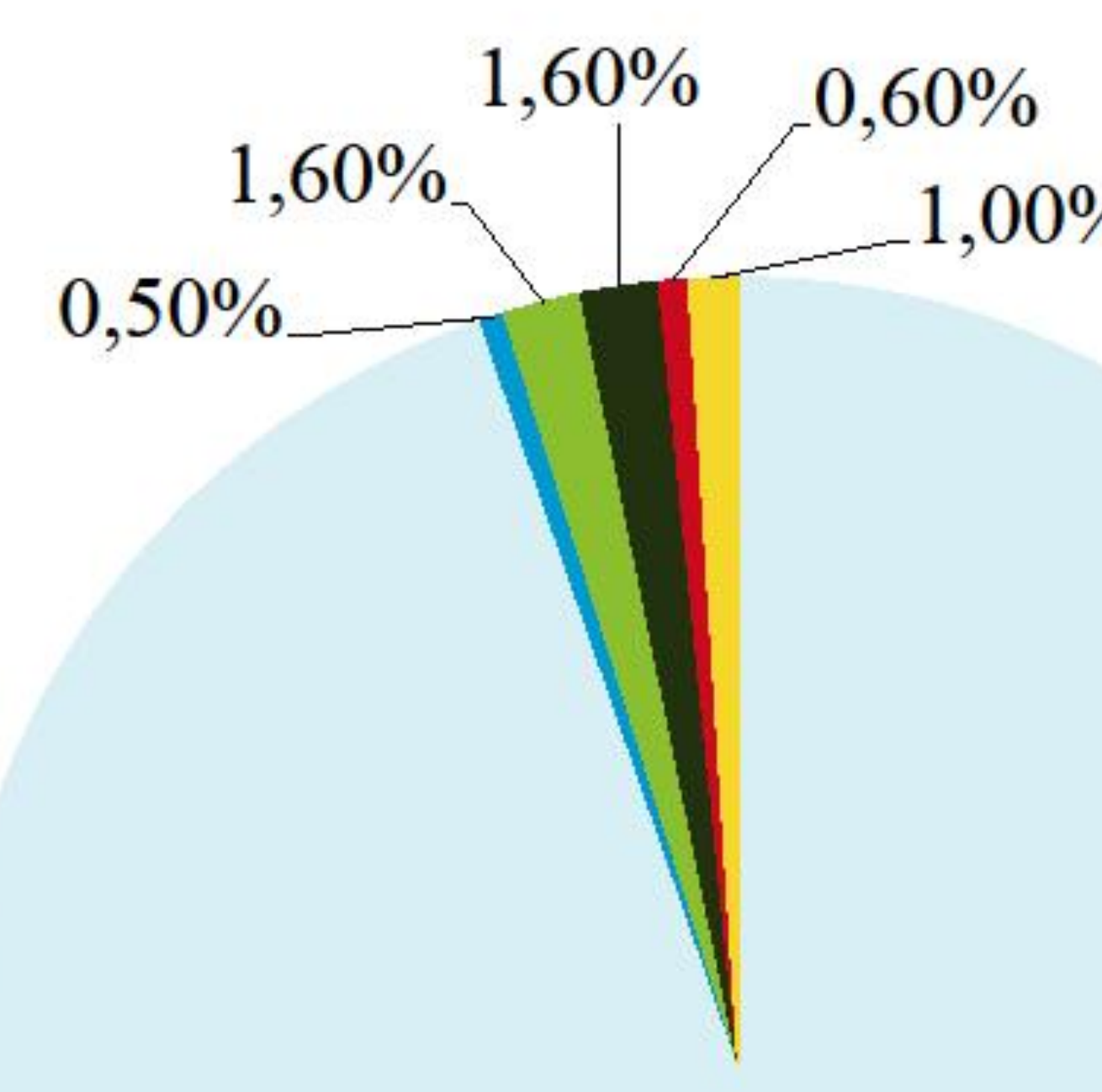

no hormonal irregularities

Hyperandrogenemia

prim. Hypogonadism

nec. Hypogonadism

n isolated high FSH

$94,30 \%$

high estradiol

\section{References}

Klinefelter Syndrome-A Clinical Update, Groth KA et al., J Clin Endocrinol Metab. 2013; 98(1):20-30

Twenty years of endocrinologic treatment in transsexualism: analyzing the role of chromosomal analysis and hormonal prifiling in the diagnostic work up, Auer MK et al. Fertility and Sterility 2013; 100(4):1103-10

Hypersexuality, Paraphilic Behaviors, and Gender Dysphoria in Individuals with Klinefelter's Syndrome, Fisher AD et al., J Sex Med 2015; 12:2413-24

\section{Discussion:}

KS affects about 1 in 660 men and is the most frequent chromosomal aberration in males, but remains often undetected with only about $25 \%$ of patients receiving the correct diagnosis. Age at diagnosis is around $35 \mathrm{yrs}$. The phenotype is thought to be linked to non-inactivated genes from the extra X-chromosome, but alternative mechanisms are possible.

The excess morbidity and mortality (expected lifespan reduced by 1.5-2 yr) may be explained by endocrine dysfunction and diseases of the cardiovascular/respiratory systems and malignancies.

Besides there are some socioeconomic differences to the healthy population (shorter education, lower income etc.) also an increased risk for criminal activity was found (crime rate for sexual abuse and arson). Only few studies have examined the association of KS and transsexuality. The prevalence of chromosomal abnormalities and disorders of sexual development (DSD) in MtF individuals varies between 1:11'900 to 1:45'000. In a retrospective study in $83 \mathrm{MtF}$ only 1 was found with primary hypogonadism $(0.5 \%)$ and normal karyotype (Figure 1 ), one was found to be KF. The study by Fisher et al. showed besides other findings a higher rate of Gender Dysphoria in KS, which was mediated by the presence of autistic traits.

Yet, no significant increase in KS was reported in previous studies looking at MtF transgender populations. Still, whether or not KS is associated with MtF has to our knowledge not systematically been investigated.

\section{Conclusion}

In MtF Individuals further work-up towards KS should be envisaged if there is additional clinical suspicion - such as clues from patient history, clinical examination (small testes) or lab work (hypergonadotropic hypogonadism) as found in our case.
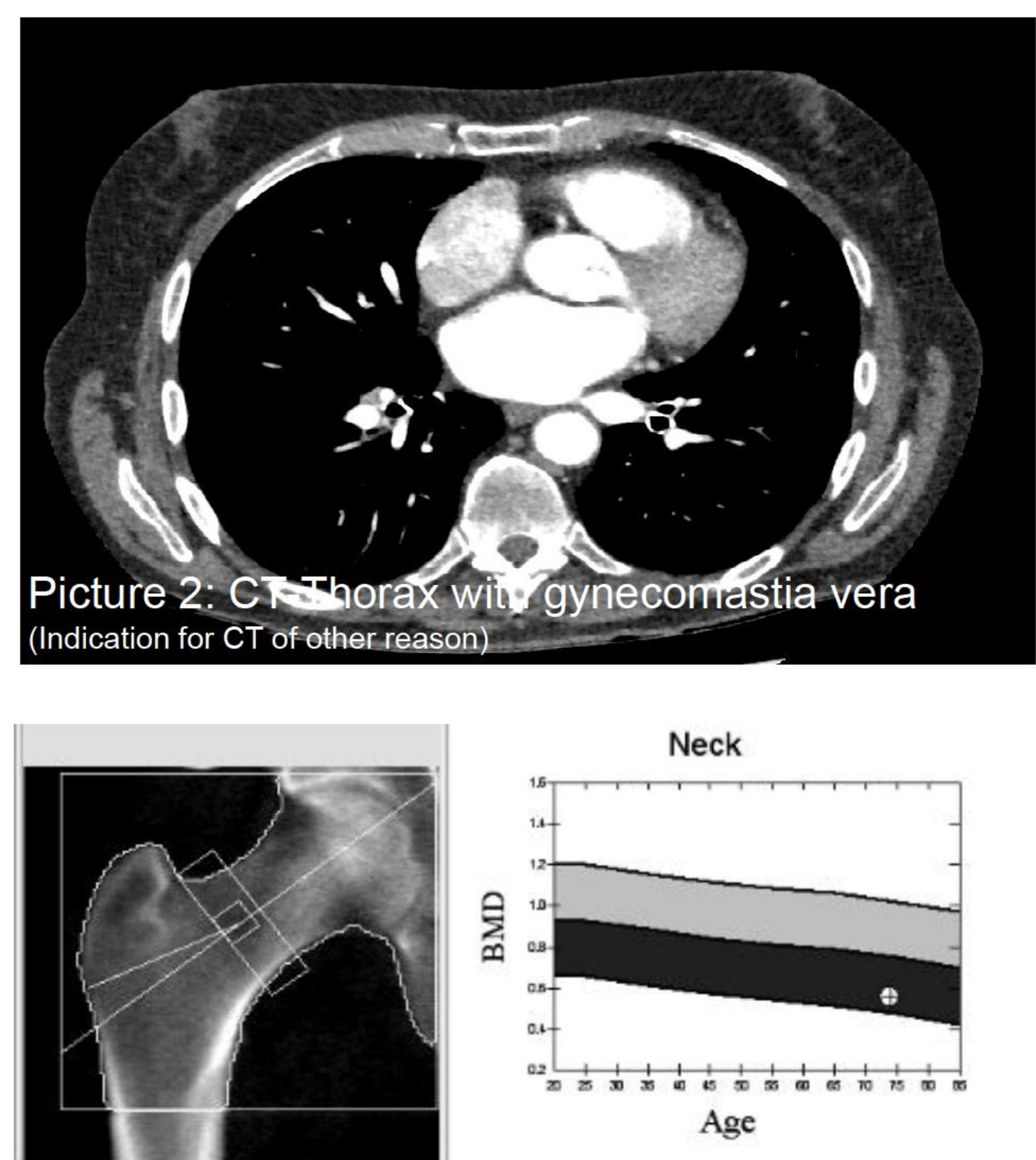

Age

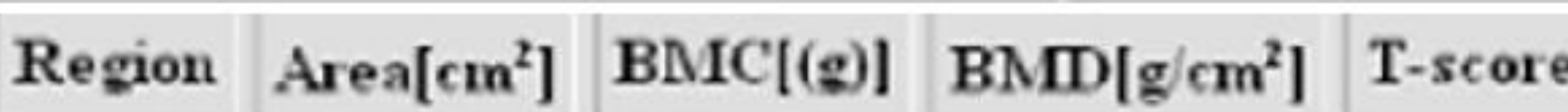

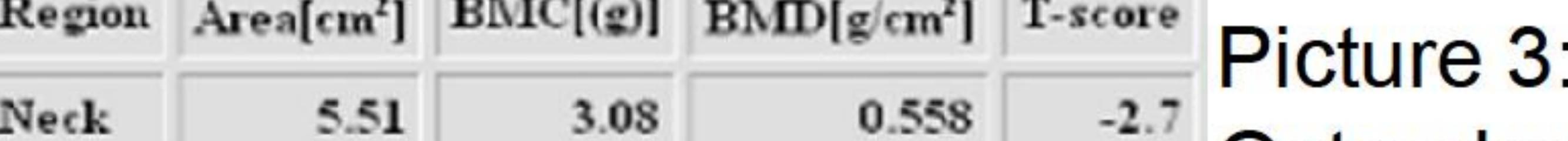

\begin{tabular}{|l|r|r|r|r|r|}
\hline Total & 39.80 & 28.69 & 0.721 & -2.1 & Osteodensitometry \\
\hline
\end{tabular} 